\title{
STANHOPEINAE MESOAMERICANAE, $V$. EL AROMA FLORAL DE LAS STANHOPEAS DE MEXICO
}

\author{
GÜNTER GERLACH \\ Botanischer Garten München-Nymphenburg, Menzinger Str. 65, 80638 München, Deutschland \\ gerlach@extern.lrz-muenchen.de
}

\begin{abstract}
Analyses of the floral fragrances of 12 of the 13 species of Stanhopea from México are presented here. Some of the species, such as S. hernandezii, S. martiana, S. tigrina, S. intermedia and S. graveolens are well characterized by their floral fragrances, while the other two species $S$. ruckeri and $S$. intermedia have two chemotypes. Furthermore, there exist two groups of 3 species each which are not delimited by their floral fragrances, just as their taxonomy and morphology also are not easily described and clear. .

Resumen. Se presentan análisis de aromas florales de 12 de las 13 especies de Stanhopea de México. Algunas, como $S$. hernandezii, S. martiana, S. tigrina, S. intermedia y S. graveolens, se pueden caracterizarar muy fácilmente por su olor, mientras que otras dos especies, $S$. ruckeri y $S$. intermedia, presentan dos quimotipos. Además, existen dos grupos con tres especies cada uno que no se delimitan por sus aromas; igual, su taxonomía y morfología no es fácil y clara.
\end{abstract}

Palabras clave / Key words : Orchids, Orquídeas, Stanhopea, México, floral fragrances, aromas florales

Este artículo está dedicado a Miguel Ángel Soto Arenas quien falleció victima de un asesinato. Miguel era un gran amante de las Stanhopea y por esta razón contribuyó bastante al conocimiento de este género. Él no solamente es autor de dos especies de Stanhopea de Mexico (Stanhopea dodsoniana Salazar \& Soto Arenas, Stanhopea whittenii Soto Arenas, Salazar \& G.Gerlach), sino también, ha visto y colectado la gran mayoría de ellas en su habitat natural. Sin él mi parte para la Flora Mesoamericana hubiera sido muy pobre, porque él puso a mi disposición los datos basicos de las especies de Stanhopea de México. Pensé en publicar este trabajo con los aromas florales de las Stanhopea de México al terminar de investigar todas las especies, pero la muerte de Miguel me impidió de hacerlo; ahora sin incluir la $S$. maculosa, la cual todavia no ha florecido en el Jardin Botánico de Munich, he decidido publicar el trabajo prematuramente.

\section{Indroducción}

El género Stanhopea está presente en México con 13 especies aqui reconocidas (Tab. 1). Todos pertenecen al subgénero Stanhopea y aquí a las secciones Saccata y Wardii. México representa un segundo centro de diversidad del género; el otro es en Colombia con 19 especies de los 3 subgéneros y en las 4 secciones. Con Stanhopea maculosa, la subtribu Stanhopeinae encuentra su límite norte de distribución en el Edo. de Sonora, en los bosques de encinos y pinos en un habitat con un período de sequía muy pronunciado. Todo el resto de las especies de Stanhopea de México se encuentra meridional del Trópico de Cáncer, en climas calientes o templados, en bosques y selvas húmedas.

Las especies de Stanhopea de México según Gerlach (1999) se agrupan en:

\section{Sección Saccata}

$S$. hernandezii $[=S$. cavendishii Lindl. ex W.Baxter, $S$. devoniensis Lindl., S. expansa P.N.Don, S. lyncea (Lindl.) P.N.Don]

S. intermedia Klinge (= S. novogaliciana S.Rosillo)

S. maculosa Knowles \& Westc. (= S. fregeana Rchb.f., S. marshii Rchb.f., S. schilleriana Rchb.f.)

S. martiana Lindl. (= S. implicata Westc. ex Lindl., $S$. velata C.Morren)

S. pseudoradiosa Jenny

${ }^{1}$ La lista de sinónimos no está completa. Se mencionan solamente los más conocidos y usados. 
TABLA 1. Lista de plantas investigadas.

\begin{tabular}{|c|c|c|c|}
\hline Especie & Numero & Origen & Elevación \\
\hline S. dodsoniana & $99 / 3327$ & $\begin{array}{l}\text { Edo. Chiapas, weniger als } 10 \mathrm{~km} \text { vor Ocosingo aus } \\
\text { Richtung San Cristobal }\end{array}$ & \\
\hline S. graveolens & $02 / 2290$ & Edo. Chiapas, Lagunas de Monte Bello & \\
\hline S. hernandezii & $92 / 3098$ & Edo. México, Toluca & \\
\hline S. intermedia & $99 / 3313$ & $\begin{array}{l}\text { Guerrero, Atoyac de Alvarez, } 6 \text { millas de El Pareiso } \\
\text { hacia Atoyac; sobre el camino Atoyac-Xochipala }\end{array}$ & $1100 \mathrm{~m}$ \\
\hline S. intermedia & Soto 3131 & $\sin$ & \\
\hline S. intermedia & $00 / 3843$ & sin (S. novogaliciana) & \\
\hline S. martiana & $99 / 3315$ & $\begin{array}{l}\text { Edo. Oaxaca, Km } 170 \text { de la carretera Oaxaca-Pto. } \\
\text { Escondido }\end{array}$ & $1840 \mathrm{~m}$ \\
\hline S. martiana & $99 / 3317$ & Edo. Jalisco (sin datos precisos) & \\
\hline S. oculata & $73 / 577$ & Guatemala (sin datos precisos) & \\
\hline S. pseudoradiosa & $99 / 3316$ & $\begin{array}{l}\text { Edo. Oaxaca, Dto.Juquila, km } 12.2 \text { de la brecha de } \\
\text { Luz de Luna a Miahuatlán; que conecta las } \\
\text { carreteras } 131 \text { y } 175\end{array}$ & $1250 \mathrm{~m}$ \\
\hline S. pseudoradiosa & $08 / 0915$ & $\sin$ & \\
\hline S. ruckeni & Soto 5115 & $\sin$ & \\
\hline S. ruckeri & $99 / 3300$ & $\begin{array}{l}\text { Edo. Chiapas, Ocosingo, Crucero Corozal, } 144 \mathrm{~km} \text { al } \\
\text { SE de Palenque; por la carretera a Marquéz de } \\
\text { Comillas }\end{array}$ & $150 \mathrm{~m}$ \\
\hline S. radiosa & $99 / 3302$ & Edo. Nayarit (sin localidad precisa) & \\
\hline S. radiosa & $99 / 3319$ & $\begin{array}{l}\text { Edo. Sinaloa, km } 223 \text { carretera Durango-Mazatlán, } \\
\text { cerca de Potrerillos }\end{array}$ & $1350-1400 m$ \\
\hline S. radiosa & $99 / 3339$ & $\sin$ & \\
\hline S. saccata & $99 / 3323$ & Edo. Chiapas, km 30 del carretera Huixtla-Motozintla & $1000 \mathrm{~m}$ \\
\hline S. tigrina & $98 / 2068$ & Edo. Tamaulipas, Gómez Farias & \\
\hline S. tigrina & $07 / 09 / 90$ & $\sin$ & \\
\hline S. tigrina & $10 / 16 / 96$ & $\sin$ & \\
\hline S. whittenii & $99 / 3306$ & $\begin{array}{l}\text { Edo. Chiapas, Municipio: Comitán de Dominguez, km } \\
\text { 11; camino al ejido de Las Margaritas }\end{array}$ & $1500 \mathrm{~m}$ \\
\hline
\end{tabular}

S. radiosa Lem.

S. saccata Batem.

S. tigrina Batem. ex Lindl. $[=S$. nigroviolacea (C.Morren) Beer]

\section{Sección Wardii}

S. dodsoniana Salazar \& Soto Arenas

$S$. graveolens Lindl. (= S. aurata Beer, $S$. venusta Lind1.)

S. oculata (= S. minor Schltr., S. purpusii Schltr.)

S. ruckeri Lindl. (= S. inodora Lodd. ex Lindl.)

S. whittenii Soto Arenas, Salazar \& G.Gerlach

\section{Materiales y Métodos}

Muestras de las plantas investigadas están depositadas en el Herbario del Botanische Staatssammlung München (M). Las flores están conservadas en líquido en una solución de agua / alcohol etílico / formol / ácido acetico / glicerina (40/45/5/5/5).

Para la colecta del aroma floral, la planta era observada en la mañana del primer dia de su floración. La inflorescencia era puesta en un recipiente de vidrio de un tamaño adecuado. El recipiente se tapaba con una hoja de aluminio. El aire cargado del perfume floral se succionaba con una bomba pequeña (personal air sampler) atraversado por un filtro de carbotrap por 3 a 4 horas (headspace technique). Después las sustancias absorbidas por el filtro fueron diluidas en hexano de alto grado de pureza e injectadas en un chromatografo de gases. La identificación de ellas fue hecha con un espectrómetro de masas y con su tiempo de retención en una columna polar (WAX fase). Todos los análisis eran realizados por R. Kaiser en el laboratorio de Givaudan en Dübendorf, Suiza (Fig. 1-2). 


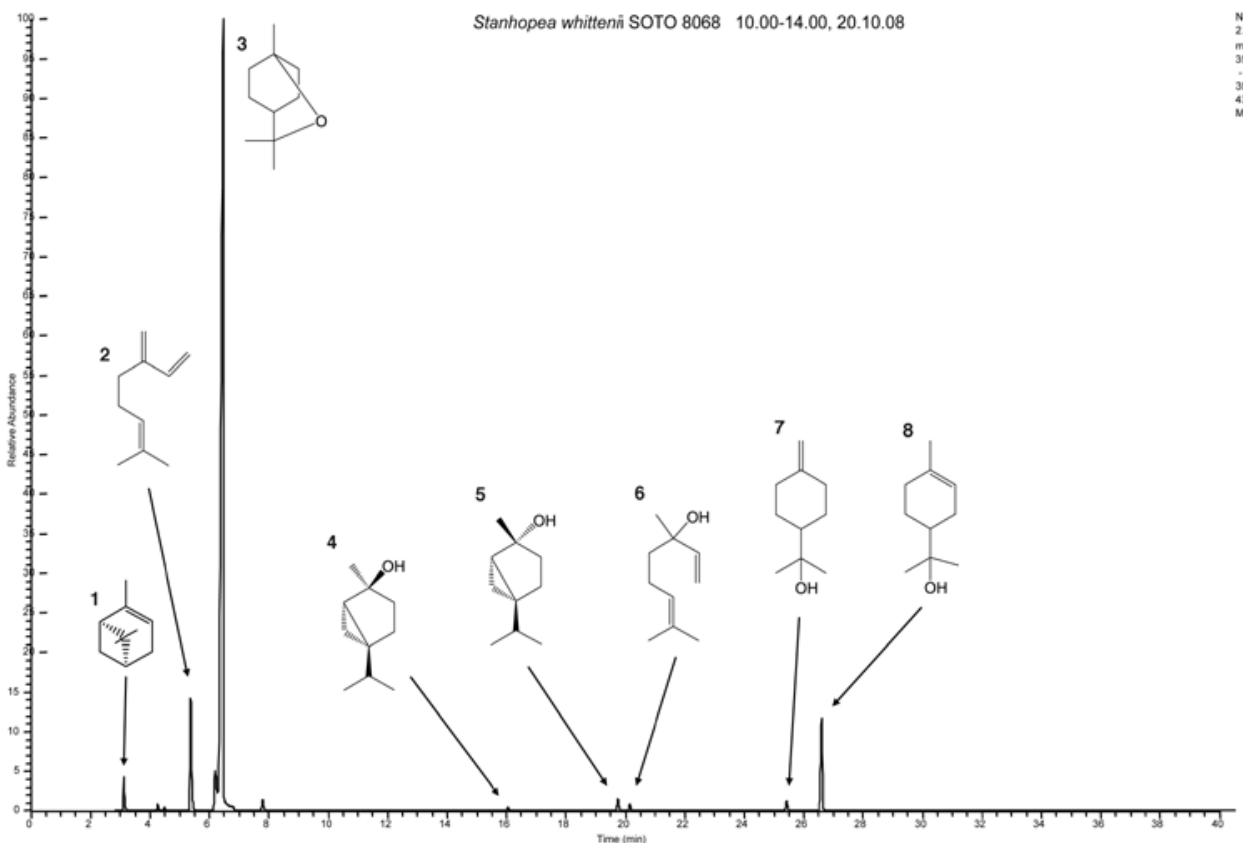

12.11.2008 13:23:01

FIgURA 1. Chromatograma del aroma floral de S. whittenii SOTO 8068. 1 - $\beta$-Pinene, 2 - Myrcene, 3 - Eucalyptol, 4 - trans-Sabinene hydrate, 5 - cis-Sabinene hydrate 6 - Linalool, 7 - $\delta$-Terpineol, 8 - $\alpha$-Terpineol (generosidad de R. Kaiser).

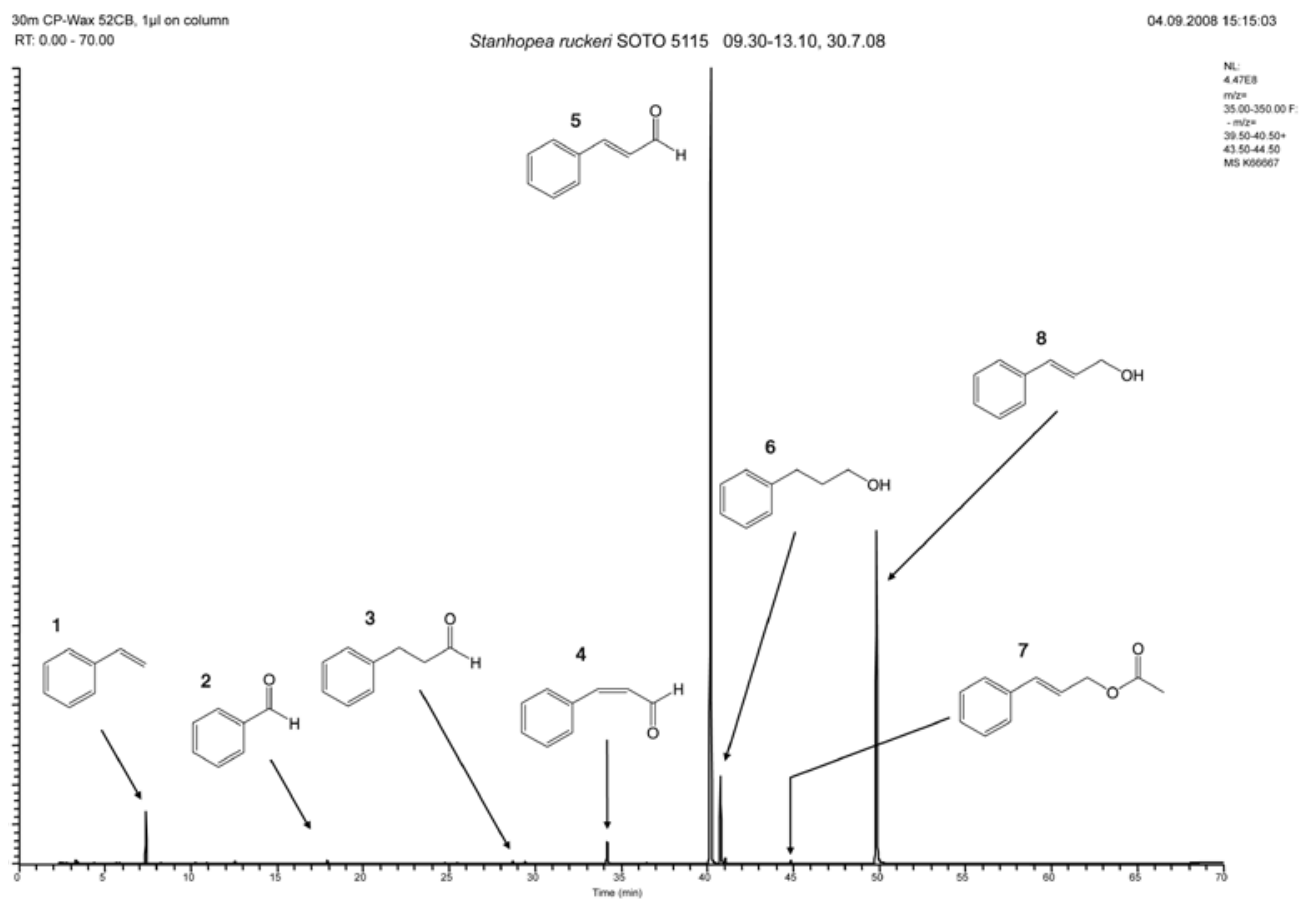

Figura 2. Chromatograma del aroma floral de S. ruckeri SOTO 5115. 1 - Styrene, 2 - Benzaldehyde, 3 - 3-Phenylpropanal, 4 (Z)-Cinnamic aldehyde, 5 - Cinnamic aldehyde, 6 - 3-Phenylpropanol, 7 - (E)-Cinnamyl acetate, 8 - (E)-Cinnamic alcohol (generosidad de R. Kaiser). 


\begin{tabular}{|c|c|c|c|c|c|c|c|c|c|c|c|c|}
\hline & 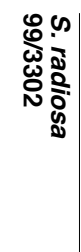 & 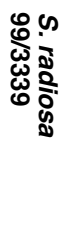 & 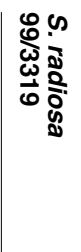 & 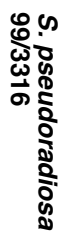 & 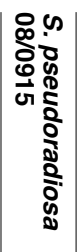 & 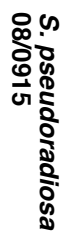 & 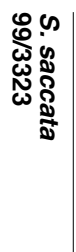 & 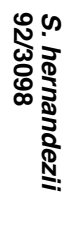 & 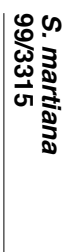 & 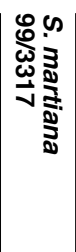 & 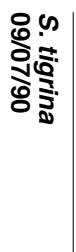 & 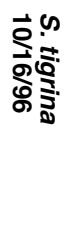 \\
\hline a-Pinene & 1.2 & 1.2 & 4 & 2.7 & 1 & 1.2 & 0.6 & 0.4 & 0.1 & 0.1 & & \\
\hline Butyl Acetate & 0.03 & & & & 0.6 & & & & & & & \\
\hline Hexanal & & & & & 0.6 & & & & & & & \\
\hline$\beta$-Pinene & 0.3 & 0.2 & 0.6 & & 0.2 & 0.2 & & 0.01 & & 0.01 & & \\
\hline Sabinene & 0.2 & 0.1 & 0.2 & 0.4 & 0.2 & & & & 0.01 & & & \\
\hline Myrcene & 1.5 & 1.2 & 2 & 1.5 & 0.6 & 0.8 & 0.2 & 0.1 & 0.1 & 0.1 & & \\
\hline Limonene & 1.4 & 0.1 & 0.5 & 1.1 & 6.2 & 0.8 & 0.1 & 0.1 & 0.03 & 0.04 & 0.02 & 0.02 \\
\hline Eucalyptol & 25.5 & 68.8 & 57 & 23.5 & 3.1 & 15.9 & 0.2 & 2.3 & 1 & 1.7 & 0.1 & 0.2 \\
\hline (Z)-Ocimene & 0.2 & 0.05 & 0.07 & 0.4 & 0.4 & 0.4 & 0.5 & & & & & \\
\hline (E)-Ocimene & 17.8 & 2.2 & 13 & 40 & 74.6 & 30 & 87.3 & & & & & \\
\hline \multicolumn{13}{|l|}{ Styrene } \\
\hline Hexyl acetate & & & & & & & & 0.1 & 0.01 & 0.03 & & \\
\hline \multicolumn{13}{|l|}{ Terpinolene } \\
\hline Hexanol & & & & & 0.3 & & & & & 0.01 & & \\
\hline (Z)-3(4)-Epoxy-3,7-dimethyl-1,6-octadiene & 0.6 & 1.7 & 0.8 & 1.6 & 0.1 & 1.1 & & & & & & \\
\hline Acetoine & & 0.4 & 0.2 & & & & & & & & & \\
\hline Nonanal & 0.02 & & & & 0.05 & & 0.08 & & 0.01 & 0.01 & 0.03 & \\
\hline (E)-3(4)-Epoxy-3,7-dimethyl-1,6-octadiene & 0.9 & 2.3 & 1.3 & 3.5 & 0.2 & 2 & 0.7 & & & & & \\
\hline 6,7-Epoxymyrcene (Myrcene epoxide) & 0.03 & 0.1 & 0.1 & & & & & & & & & \\
\hline p-Methylanisole & & & & 0.03 & & 0.02 & & & & & & \\
\hline Rosefuran & 0.05 & & & 0.3 & & & & & & & & \\
\hline (Z)-Ocimene epoxide & 0.1 & 0.2 & & 0.4 & & 0.2 & 0.1 & & & & & \\
\hline trans-Sabinene hydrate & & 0.1 & & & & & & & & & & \\
\hline Acetic acid & & & 2 & & & & 0.08 & & & & 0.08 & \\
\hline$\overline{(E) \text {-Ocimene epoxide }}$ & 0.9 & 1.4 & 1.3 & 4 & 0.3 & 3.1 & 1.3 & & & & & \\
\hline Decanal & 0.01 & & & & 0.05 & & 0.06 & & & & & \\
\hline Benzaldehyde & 1.8 & 1 & 2.8 & 0.6 & & 0.2 & 0.05 & 0.4 & 0.02 & 0.02 & 0.2 & 0.02 \\
\hline cis-Sabinene hydrate & 0.1 & 0.3 & 0.2 & & & & & & & & & \\
\hline Linalool & 0.2 & 0.5 & 0.2 & 0.2 & 0.2 & 0.2 & 0.1 & 0.1 & 29.7 & 7 & 0.05 & 0.02 \\
\hline Octanol & 0.01 & & & & & & 0.2 & & & & & \\
\hline Terpinen-4-ol & & & & & & & & & & & & \\
\hline Methyl benzoate & 2.9 & 0.6 & 1 & 0.9 & 0.1 & 0.1 & & 0.5 & 0.4 & 0.2 & 0.9 & 0.03 \\
\hline \multicolumn{13}{|l|}{ cis- $\beta$-Terpineol } \\
\hline $\bar{\delta}$-Terpineol & 0.4 & & 0.1 & & & & & & & & & \\
\hline$\overline{\mathrm{a}-T e r p i n e o l}$ & 0.9 & 1.6 & 1.1 & & & 0.2 & & & 0.01 & 0.01 & & 0.05 \\
\hline Benzyl acetate & & & & & & & & 11.6 & 0.02 & 0.04 & 1.4 & 3.2 \\
\hline$(\mathrm{E}, \mathrm{E})$-a-Farnesene & & & & & & & & 13 & 0.5 & 0.2 & & \\
\hline 1,4-Dimethoxybenzene & 0.02 & 0.1 & & & & 0.6 & & & & & & \\
\hline Methyl salicylate & 0.07 & 0.1 & 0.1 & 0.2 & & & & 0.6 & 1.1 & 3 & 1.6 & 0.2 \\
\hline Phenylethyl acetate & & & & & & & & & 63.3 & 86 & 93.5 & 90.1 \\
\hline$\overline{(E, E)-2,6-D i m e t h y l-3,5,7-o c t a t r i e n-2-o l ~}$ & 0.2 & & & & 0.05 & & 0.6 & & & & & \\
\hline Methyl 3-phenylpropionate & 0.9 & & & & & 0.2 & & & & & & \\
\hline Benzyl alcohol & 0.3 & 0.2 & 0.6 & 0.1 & & & & 0.2 & 0.01 & 0.01 & 0.1 & 0.1 \\
\hline (Z)-Cinnamic aldehyde & & 0.4 & 0.2 & & & 0.1 & & & & & & \\
\hline Phenylethyl alcohol & & & & 1.1 & & & & & 0.3 & 0.4 & 0.7 & 0.8 \\
\hline Phenylacetonitrile & & & & & & & & 3 & & & & \\
\hline 3-Phenylpropyl acetate & 0.07 & 0.07 & 0.05 & & & & & & & & & 0.05 \\
\hline Methyl (Z)-cinnamate & 0.06 & & & & & 0.6 & & & & & & \\
\hline Cinnamic aldehyde & 9.3 & 5.4 & 6.2 & 0.03 & 0.08 & 1.5 & 0.07 & & & & & \\
\hline 3-Phenylpropanol & 1.8 & 1.2 & 2 & & & & & & & & & \\
\hline \multicolumn{13}{|l|}{ (E)-Nerolidol } \\
\hline Methyl (E)-cinnamate & 26.4 & & & & 0.3 & 35 & & & & & & \\
\hline (E)-Cinnamyl acetate & & & & & & & & 64 & & & & \\
\hline Methyl anthranilate & & & & & & & & 0.02 & 0.05 & & & 0.02 \\
\hline (E)-Cinnamic alcohol & 0.06 & & & & & & 0.1 & 0.1 & & & & 4.2 \\
\hline p-Methoxyphenylethyl acetate & & & & & & & & & & & & 0.04 \\
\hline Indole & & & & & & & & 3 & 3.3 & & & 0.6 \\
\hline Vanilline & & & & & & & & & 0.02 & 0.04 & 0.2 & \\
\hline Benzyl benzoate & & & & & & & & 0.2 & & & 0.2 & \\
\hline
\end{tabular}

TABLA 2. Análisis de aromas florales. 


\begin{tabular}{|c|c|c|c|c|c|c|c|c|c|c|c|c|}
\hline & 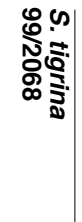 & 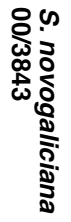 & 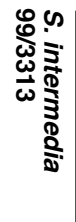 & 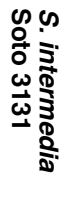 & 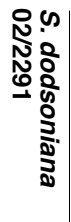 & 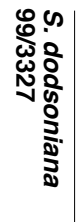 & 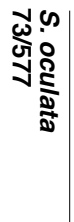 & 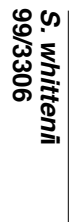 & 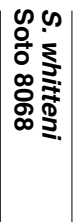 & 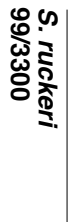 & 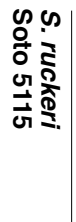 & 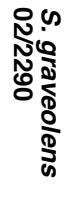 \\
\hline a-Pinene & 0.1 & 1.4 & 2 & 1.7 & 0.9 & 3.8 & & 2.1 & 1.5 & 0.3 & & 2.8 \\
\hline \multicolumn{13}{|l|}{ Butyl Acetate } \\
\hline Hexanal & & & & & & & & & & 0.05 & & \\
\hline$\beta$-Pinene & 0.02 & 0.4 & 0.4 & 0.4 & 0.3 & 1 & 0.3 & 0.4 & 0.3 & & & 0.7 \\
\hline Sabinene & & 0.2 & 0.3 & 0.4 & 0.2 & 0.6 & 0.2 & 0.3 & 0.2 & 0.1 & & 0.5 \\
\hline Myrcene & 0.04 & 9.2 & 6.8 & 6 & 6.8 & 6.7 & 3.6 & 7.5 & 7 & 0.04 & & 11.4 \\
\hline Limonene & & 2 & 2.7 & 3.5 & 4.3 & 3 & 0.5 & 4 & 3.6 & 0.5 & 0.04 & 7 \\
\hline Eucalyptol & 0.8 & 75 & 48.5 & 65.5 & 67.5 & 75 & 68 & 81.8 & 76.9 & 0.1 & & 49 \\
\hline (Z)-Ocimene & & 0.02 & & 0.01 & 0.02 & 0.01 & 0.1 & & 0.01 & & & \\
\hline (E)-Ocimene & 0.01 & 0.7 & 0.5 & 0.3 & 0.7 & 0.4 & 0.4 & 0.4 & 0.7 & & & \\
\hline Styrene & & & & & & & & & & 0.07 & 2.8 & \\
\hline Hexyl acetate & 0.04 & & & & & & & & & & & \\
\hline Terpinolene & & 0.06 & 0.04 & 0.02 & 0.03 & 0.04 & 0.05 & & & & & \\
\hline Hexanol & & & & 0.04 & & & & & & 0.1 & 0.1 & \\
\hline \multicolumn{13}{|l|}{ (Z)-3(4)-Epoxy-3,7-dimethyl-1,6-octadiene } \\
\hline Acetoine & & & & & & & & & & & 0.04 & \\
\hline Nonanal & & 0.02 & & & 0.01 & 0.01 & & 0.03 & & 0.07 & 0.2 & \\
\hline \multicolumn{13}{|l|}{ (E)-3(4)-Epoxy-3,7-dimethyl-1,6-octadiene } \\
\hline 6,7-Epoxymyrcene (Myrcene epoxide) & & 0.04 & & 0.02 & 0.02 & 0.02 & & 0.05 & 0.02 & & & 0.05 \\
\hline p-Methylanisole & & & & 0.01 & & & & & & 0.03 & & \\
\hline \multicolumn{13}{|l|}{ Rosefuran } \\
\hline \multicolumn{13}{|l|}{ (Z)-Ocimene epoxide } \\
\hline trans-Sabinene hydrate & & 0.2 & 0.2 & 0.1 & 0.2 & 0.2 & 0.2 & 0.2 & 0.2 & & & 0.7 \\
\hline \multicolumn{13}{|l|}{ Acetic acid } \\
\hline \multicolumn{13}{|l|}{ (E)-Ocimene epoxide } \\
\hline Decanal & & & & & & 0.01 & & & & & 0.04 & \\
\hline Benzaldehyde & 0.01 & 0.2 & 0.2 & 0.2 & & & & & & 0.2 & 0.2 & \\
\hline cis-Sabinene hydrate & & 0.6 & 0.5 & 0.2 & 0.9 & 0.6 & 0.2 & 0.2 & 0.8 & & & 1.8 \\
\hline Linalool & 0.04 & 0.2 & 0.4 & 0.2 & 0.5 & 0.3 & 0.4 & 0.04 & 0.5 & 0.03 & & 1.3 \\
\hline Octanol & & & & & & & & & & 0.03 & 0.04 & \\
\hline Terpinen-4-ol & & & 0.03 & 0.01 & 0.02 & 0.01 & 0.2 & 0.03 & 0.03 & 0.04 & & 0.1 \\
\hline Methyl benzoate & & 0.3 & 0.9 & 0.03 & & & & & & & & \\
\hline cis- $\beta$-Terpineol & & 0.04 & 0.04 & 0.02 & 0.08 & 0.05 & 0.4 & 0.03 & 0.05 & & & 0.1 \\
\hline $\bar{\delta}$-Terpineol & & 0.5 & 0.7 & 0.3 & 1.2 & 0.6 & 1 & 0.08 & 0.7 & & & 1.3 \\
\hline a-Terpineol & 0.07 & 7 & 5.3 & 2.4 & 15.1 & 6.8 & 13.7 & 1.1 & 7.1 & & & 10.6 \\
\hline Benzyl acetate & 2 & & 0.4 & 0.1 & & 0.05 & & & & & & \\
\hline$(\mathrm{E}, \mathrm{E})$-a-Farnesene & & & 12 & 7.8 & & & & & & & 0.2 & 1.1 \\
\hline \multicolumn{13}{|l|}{ 1,4-Dimethoxybenzene } \\
\hline Methyl salicylate & 0.2 & & & & & & & & & & & \\
\hline Phenylethyl acetate & 87 & & 2.3 & 3.1 & & 0.01 & & 1.5 & & 2.7 & 0.01 & 0.2 \\
\hline \multicolumn{13}{|l|}{$(\mathrm{E}, \mathrm{E})-2,6$-Dimethyl-3,5,7-octatrien-2-ol } \\
\hline \multicolumn{13}{|l|}{ Methyl 3-phenylpropionate } \\
\hline Benzyl alcohol & & 0.1 & 0.3 & 0.2 & & & & 0.01 & & 0.1 & 0.01 & \\
\hline (Z)-Cinnamic aldehyde & & & & & & & & & & & 1.6 & \\
\hline Phenylethyl alcohol & 1.5 & & 0.2 & 0.5 & & & & 0.01 & & 90.3 & & \\
\hline \multicolumn{13}{|l|}{ Phenylacetonitrile } \\
\hline 3-Phenylpropyl acetate & & & & & & & & & & & 0.07 & \\
\hline \multicolumn{13}{|l|}{ Methyl (Z)-cinnamate } \\
\hline Cinnamic aldehyde & & & & & & & & & & & 61 & \\
\hline 3-Phenylpropanol & & & & & & & & & & & 6.4 & \\
\hline (E)-Nerolidol & & & 0.01 & 0.01 & & & & & & 0.08 & 0.5 & 0.03 \\
\hline \multicolumn{13}{|l|}{ Methyl (E)-cinnamate } \\
\hline (E)-Cinnamyl acetate & 6.4 & & & 0.01 & & & & & & & 0.2 & \\
\hline Methyl anthranilate & & & 0.1 & 0.1 & & & & 0.01 & & & & \\
\hline (E)-Cinnamic alcohol & 0.06 & & & & & & & & & & 25 & \\
\hline p-Methoxyphenylethyl acetate & 0.05 & & 0.4 & 0.07 & & & & & & & & \\
\hline Indole & 1.4 & & 13 & 6.3 & 0.6 & & 9.2 & & & & & 10 \\
\hline Vanilline & & & & & 0.2 & 0.03 & 0.2 & & & & & \\
\hline Benzyl benzoate & & & & & & & 0.2 & & 0.01 & & & \\
\hline
\end{tabular}

TABLA 2. Análisis de aromas florales. 


\section{Resultados y Discusión}

No todas las sustancias presentadas en la Tabla 2 tienen importancia en la atracción de los polinizadores, pero en la mayoría de los casos no sabemos si son percibibles por estos animales o no. Hay muchas sustancias que son comunitarias como mencionan también Whitten \& Williams (1992), entre estas especialmente los monoterpenos ( $\alpha$-pineno, $\beta$-pineno, sabineno, mirceno, limoneno, eucaliptol $(1,8$-cineol) y los ocimenos). Sabemos que entre ellos hay unos de los mas fuertes atrayentes para los euglosidos, pero como estan presentes en casi todas las especies no pueden servir como rasgos diferenciales. Como consecuencia estas sustancias no están consideradas en la discusión.

\section{SeCción SACCATA}

a) Grupo de S. saccata (S. pseudoradiosa, S. radiosa y S. saccata)

Estas tres especies forman un grupo de gran similaridad morfológica. Tienen el labelo profundamente saccato y una coloración muy parecida de amarillo hasta crema con una puntuación muy fina, pero nunca con manchas. Se diferencian especialmente en el tamaño y la forma de la apertura del hipoquilo. Jenny (2004) muestra muy bien un gradiente de aperturas en 5 especimenes de $S$. radiosa y $S$. pseudoradiosa en sus figuras 267 y 268 (p.178) ${ }^{2}$. Poniendo estos labelos con este de S. saccata (Fig. $5 \mathrm{~F}$ ) en línea uno puede observar un gradiente de anchura de las aperturas de $S$. pseudoradiosa (Fig. 3D) a $S$. radiosa (Fig. 5D) hacia $S$. saccata. Las tres especies provienen del lado pacífico de la Sierra Madre Occidental pasando por la Sierra Madre del Sur a Chiapas llegando hasta Honduras. La más norteña es $S$. radiosa, la sureña $S$. saccata. Entonces el gradiente de la apertura no se refleja en la distribución. Según Jenny las especies no crecen mixtas, pero todavía hay muy pocas observaciones para comprobar éste factor con seguridad.

Las 8 muestras de aroma publicadas por Whitten \& Williams (1992) y las 7 muestras adicionales presentadas aquí tienen (E)-ocimeno como componente significante en porcentajes entre 2,2\% y

\footnotetext{
${ }^{2}$ La foto esta invertida, o hay que cambiar izquierdo por derecho.
}

$87,3 \%$. El cinamato de metilo junto con sus derivados representa otra característica en algunos clones. En la Tabla 2 se presentan dos analisis de la misma planta de $S$. pseudoradiosa realizados en dos floraciones diferentes; es interesante ver la variabilidad del aroma floral. Quiero sumarme a la opionion de Whitten \& Williams que en este grupo empieza una especiación alopatrica.

\section{b) Stanhopea intermedia y S. novogaliciana}

Por su morfología floral las dos especies no se pueden distinguir. Conservadas en líquido y así descoloridas flores de las dos especies no se pueden diferenciar. Unicamente el color es el caracter diagnostico para las dos, S. intermedia es casi unicolor de un amarillo pálido (Fig. 4A, 5A), mientras $S$. novogaliciana tiene el color amarilla fuerte con manchas grandes marrones en las bases de los pétalos y del labelo (Fig. 5C). Rosillo (1984) publica otro argumento para la diferencia de las dos. Él dijo que las dos crecian juntas en la misma barranca, pero solamente la $S$. novogaliciana era visitada por euglosidos de color azul metálico, mientras que la $S$. intermedia, igual en flor no atraía ninguna abeja. Es frequente si uno ve varias Stanhopeinae de la misma especie en un sitio en flor que unos esten visitados fuertemente mientras que otros carecen de visitantes. Eso depende del comportamiento de los euglosidos, cuales andan frequentemente en pequeños grupos. Aparecen en una inflorecencia, pelean entre ellos por los aromas y después de cierto tiempo se van. Por esta razón supongo que era un efecto casual. Observaciones al respecto serían muy agradecidas!

La composición del aroma floral de las dos se diferencia bien. Las sustancias mayoritarias comunes de las dos son: mirceno, limoneno, eucaliptol y $\alpha$-terpineol. La $S$. novogaliciana investigada contiene mucho menos componentes en el aroma floral, le faltan el indol y el (E,E)- $\alpha$-farneseno como componentes de alta concentración. Puede ser que se trata de un artefacto en la recolección del aroma, porque el indol no es muy volatil y por esta razón se colecta con más dificultad y especialmente a temperaturas bajas. Por otro lado también se puede aclarar con la presencia de dos quimotipos que tenemos tambien en otras especies, por ejemplo en $S$. ruckeri, una especie con un concepto morfologico bien claro. 
La declaración de Rosillo (1984) del euglosido azul comparándole con el polinizador de $S$. radiosa (por el tamaño), nos guía a una especie del género Euglossa; con alta probabilidad se trata de Euglossa mixta una especie con amplia distribución. Por la morfología tan similar de ambos y el crecimiento en la misma región se hace bien visible que se trata de una sola especie que aparece en dos coloraciones diferentes y dos quimotipos. Por las reglas del código botánico hay que usar el nombre mas antiguo, así $S$. novogaliciana cae en la sinonimía de $S$. intermedia.

c) Resto de la Sección (S. hernandezii, S. maculosa, S. martiana y S. tigrina)

En la taxonomía del resto de la sección no hay problemas en la delimitación de las especies. Todas tienen el epiquilo con los margenes erectos, formando un $\mathrm{V}$ en corte transversal, asi con el ápice tridentado, con la punta central en la mayoría de las especies mas cortas que los laterales. Todos tienen manchas, a veces muy grandes y en algunos clones de $S$. tigrina ocupando todo el superficie, dejando solamente los margenes en su color basico (S. tigrina f. nigroviolacea).

Estas especies son endémicas de México, vienen de bosques mesophyticos desde los Edos. de Sonora y Tamaulipas en el norte hasta Oaxaca y Veracruz en el sur.

La composición química de la fragrancia de tres especies de este grupo fue analizado, dejando solamente $S$. maculosa sin investigación de aroma floral. El aroma floral de S. martiana (Fig. 4B) y S. tigrina (Fig. 6A) está dominado por el acetato de feniletilo, una sustancia de aroma muy fuerte y pesado. Las dos se diferencian en su aroma por tener acetato de benzilo y salicilato de metilo en $S$. tigrina y linalool en $S$. martiana en porcentajes mayores. $S$. hernandezii (Fig. 3B) tiene un aroma bien diferente de las dos, reminiscente a canela. Grandes cantidades de acetato de cinamilo y acetato de benzilo son responsable de este olor. Estas tres especies se caracterizan muy bien por su aroma floral, no haya problema de indentificarlas con este caracter.

\section{SECCIÓN WARDII}

a) Grupo de S. oculata (S. dodsoniana, S. oculata y S. whittenii)

Este grupo es el más difícil en su taxonomía entre todas las especies de Stanhopea. Las plantas vienen desde la Sierra Madre Oriental hasta Nicaragua. $S$. oculata (Fig. 3C) es conocida de los Edos. de San Luis Potosi, Puebla, Veracruz, Oaxaca y Chiapas de México llegando hasta Nicaragua en el sureste, $S$. dodsoniana (Fig. 5B) de Veracruz, Oaxaca y Chiapas y llega también a Nicaragua, mientras que $S$. whittenii (Fig. 6B) tiene su distribución desde Chiapas en México hasta Guatemala y Belice. Entonces en el Edo. de Chiapas todas las tres están presentes, $S$. dodsoniana y $S$. oculata tienen la distribución en los Edos. de Veracruz, Oaxaca y Chiapas en común. Según el aroma floral las tres se diferencian por detailles insignificantes: aunque hay unas 30 muestras de aroma investigadas, todavía no se ha detectado un carácter discriminador. Es probable que hay híbridos naturales en las muestras investigadas, porque son morfologicamente bien parecidos y hay algunos clones que son intermedias en su forma entre dos especies. Aunque estas tres especies son las mas comunes de las Stanhopeas de México todavía casi no hay datos de su polinización. Según Whitten \& Williams (1992) Dodson \& Williams observaron la polinización por Eufriesea caerulescens en Chiapas de una planta traída de un jardín de Oaxaca. Como en esta época solamente se conocía $S$. oculata, no podemos decir con seguridad si trataba verdaderamente de esta especie. Aquí unicamente la observación de la polinización y el estudio de poblaciones puede aclarar la confusión al respecto de su taxonomía.

\section{b) S. ruckeri}

Los dos clones investigados de S. ruckeri presentan un aroma totalmente diferente. Una huele muy agradable a canela (derivados de alcohol cinámico), la otra (Fig. 3E) está dominada por el alcohol y el acetato de feniletilo, así tiene un aroma pesado. Sería sumamente interesante investigar más clones de esta especie para ver si tiene dos quimotipos o si el aroma floral es muy variable.

\section{c) S. graveolens}

Esta especie (Fig. 3A) es la más fácil de distinguir de todas las Stanhopea. Su aroma es algo desagradable causado por un alto porcentaje de indol (10\%). Dos clones mas de ésta especie de Guatemala fueron investigados y muestran el mismo patrón en la composición del aroma floral. Concentraciones 


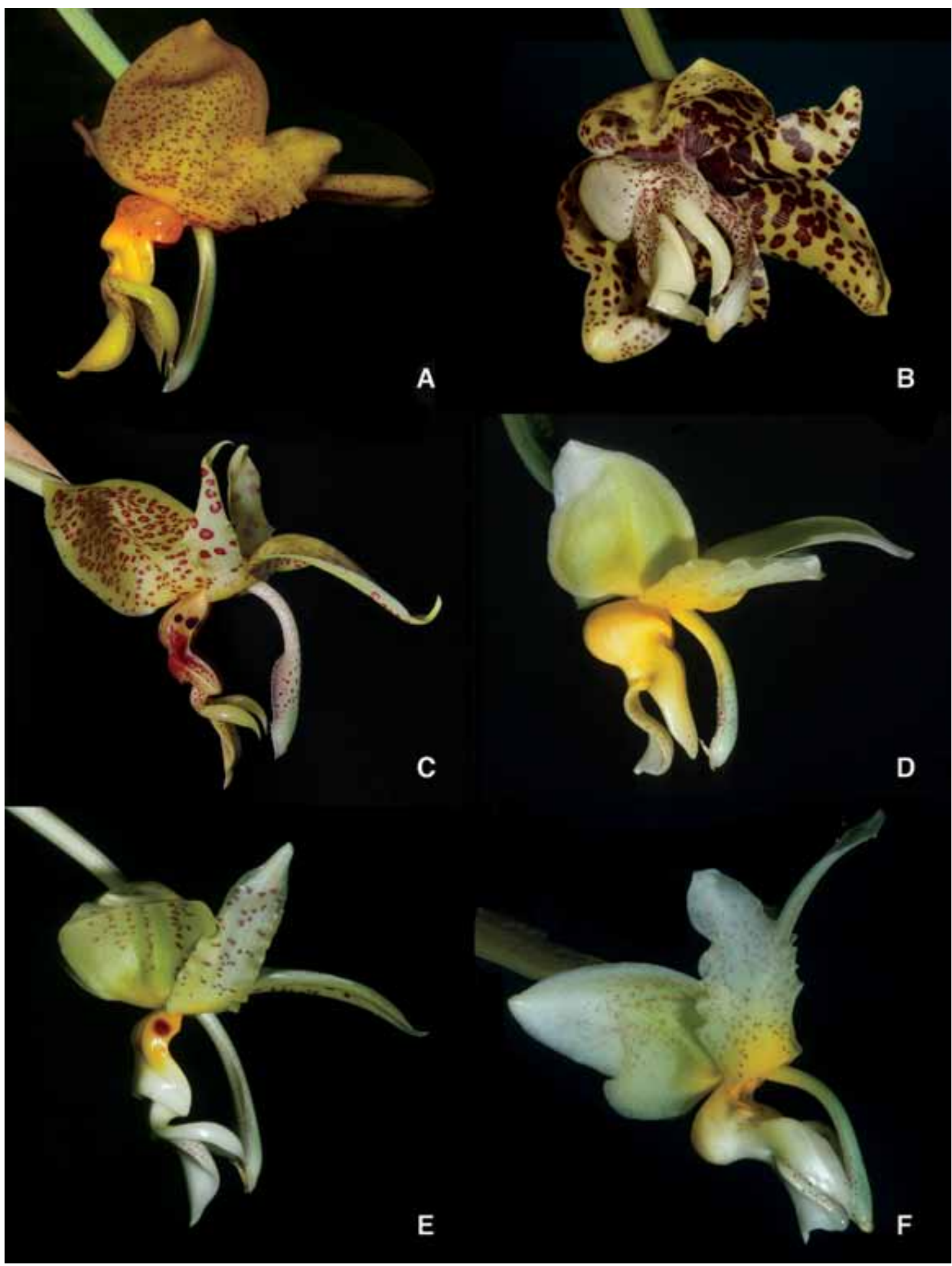

Figure 3. A - Stanhopea graveolens 02/2290. B — S. hernandezii 92/3098. C — S. oculata 73/577. D - S. pseudoradiosa 99/3316. E - S. ruckeri 99/3300. F — S. saccata 99/3323. Photo by G. Gerlach. 

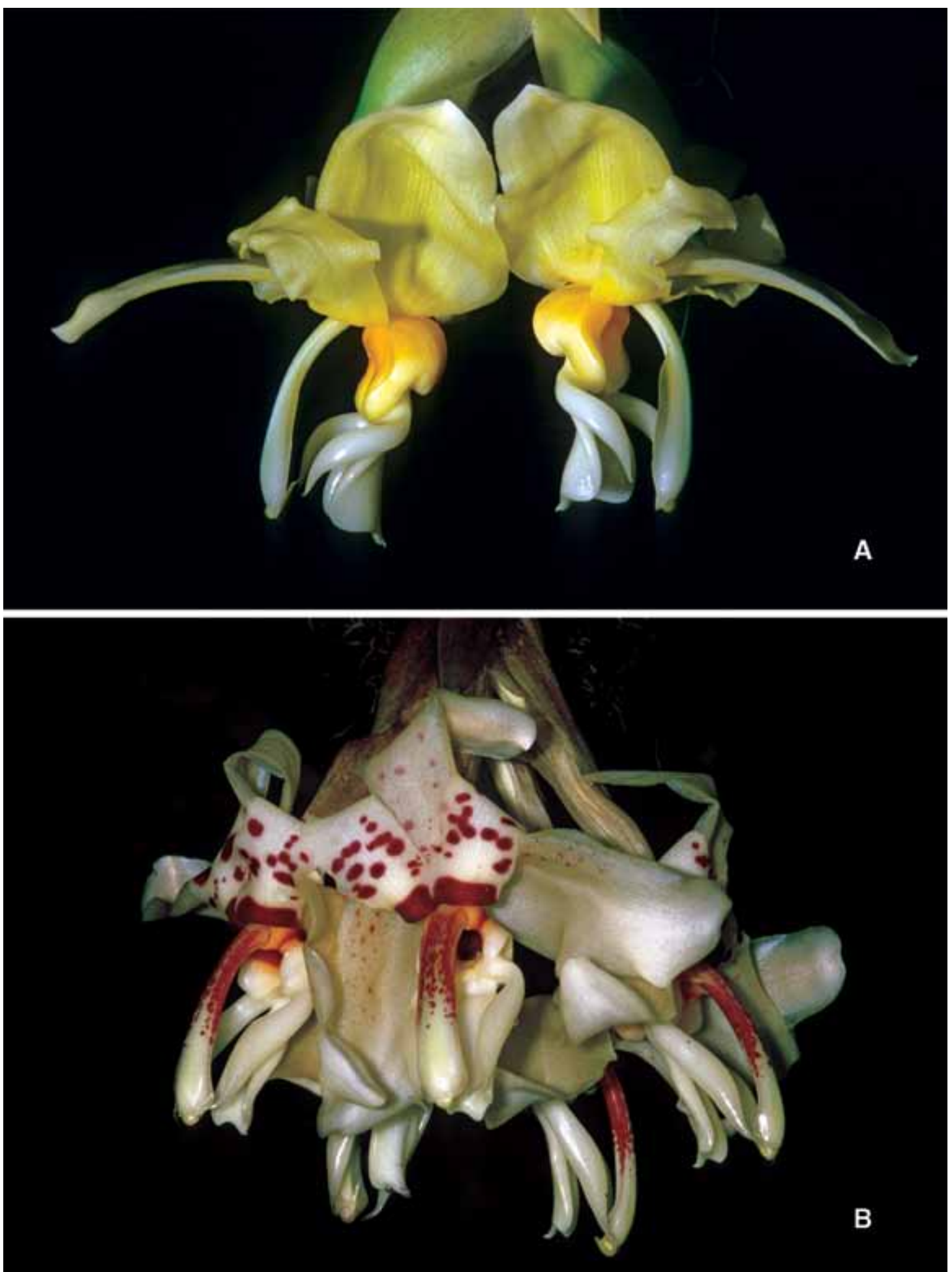

FIGURE 4. A - Stanhopea intermedia 99/3313. B - S. martiana. Photo by G. Gerlach. 


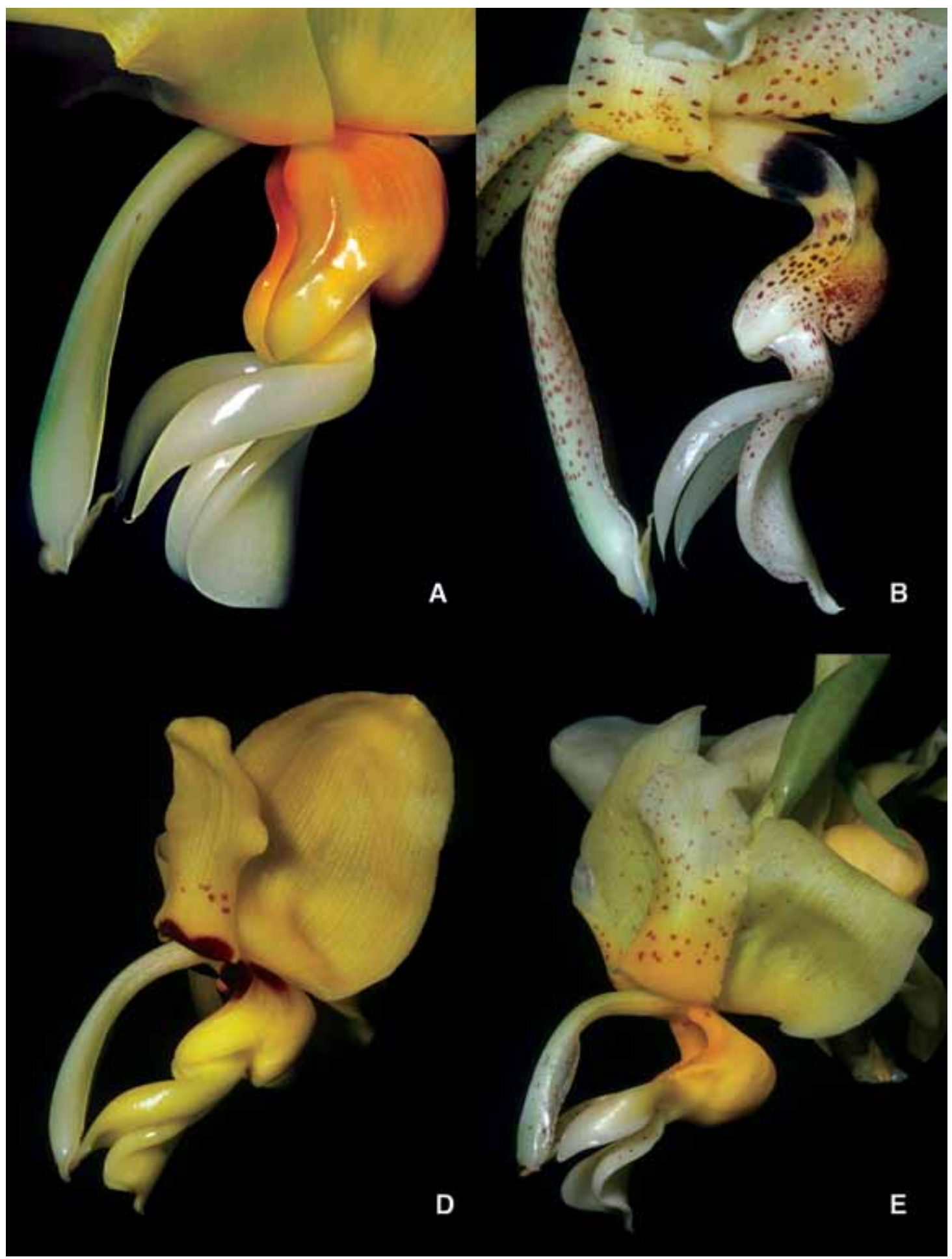

FiguRE 5. A - Stanhopea intermedia 99/3313. B - S. dodsoniana 99/3327. C - S. novogaliciana 00/3843. D - S. radiosa 99/3302. Photo by G. Gerlach. 

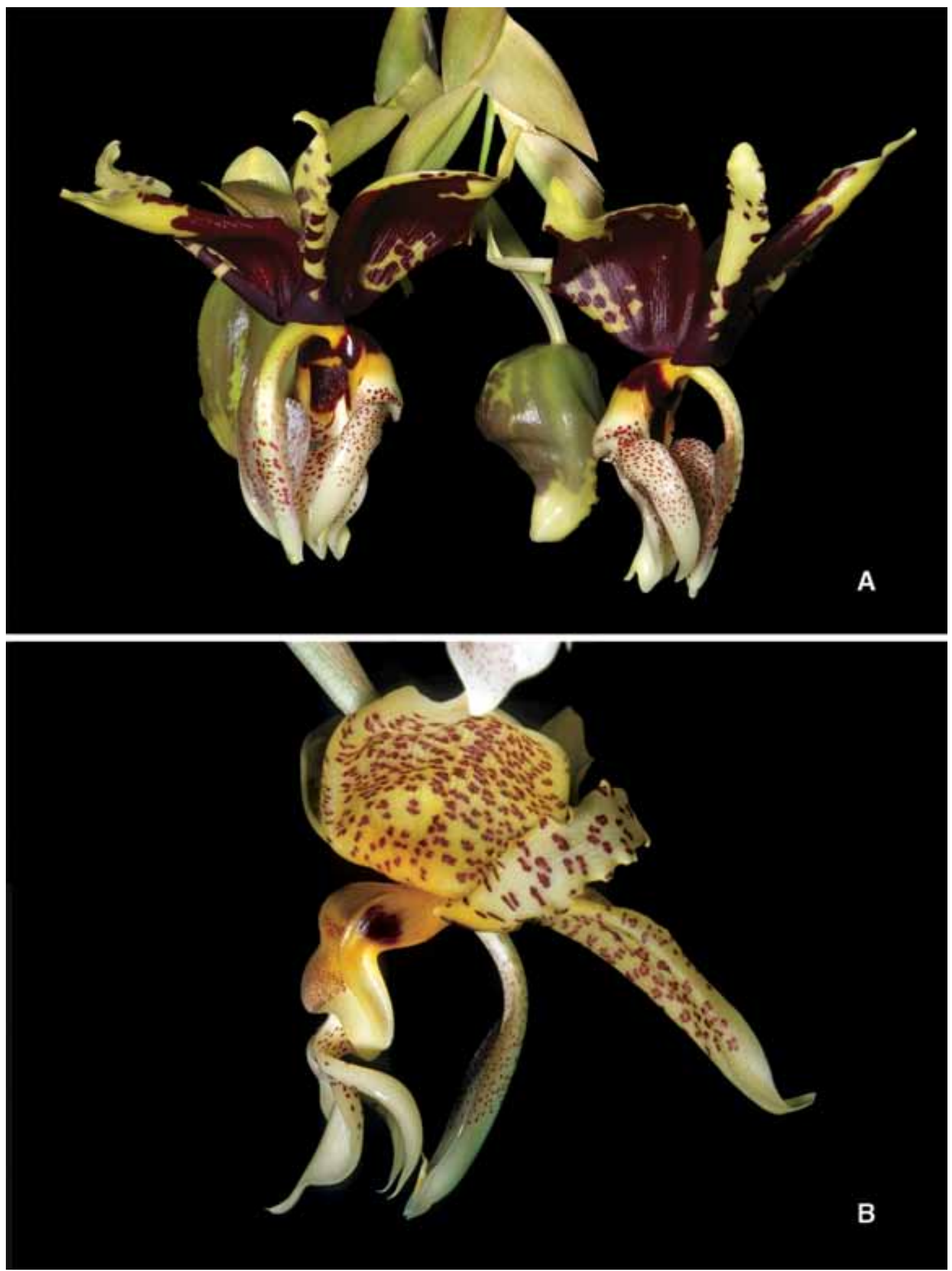

FIGURE 6. A - Stanhopea tigrina 99/2068. B - S. whittenii 99/3306. Photo by G. Gerlach. 
similares de indol se encuentran además en $S$. hernandezii, S. intermedia y S. martiana. El aroma de $S$. graveolens casi no se puede distinguir del de $S$. intermedia.

\section{Conclusiones}

Tomando en cuenta que los polinizadores de las especies de Stanhopea son solamente Euglósidos en la búsqueda de aromas preferidos hay que echar el ojo especialmente a este carácter. Los análisis de las aromas florales muestran que algunas especies están caracterizadas por el aroma como $S$. hernandezii, $S$. martiana, S. tigrina, $S$. intermedia y $S$. graveolens, mientras que otras presentan dos quimotipos o son bastante variable en su composición.

Lamentablemente las investigaciones de aromas florales están restringidas a un laboratorio y la colección del aroma floral en el campo es bastante difícil. La taxonomía está basada en muestras de herbario y descripciones que no incluyen informaciones de la fragancia de la respectiva especie. La dificultad es que son dos disciplinas que no cuadran, en el campo y en el herbario no se puede analizar los aromas, solamente al revés funciona, a tener la planta en flor uno puede hacer el análisis del aroma floral y cuadrarlo con el polinizador y la taxonomía. Este método consume mucho tiempo, así hay pocos colegas que investigan en esta dirección. Observar plantas en el campo en muchas ocasiones es un caso de suerte, primero la floración es muy corta ( 2 a 4 días), segundo la ó las plantas observadas deben de estar al alcance del observador y no en el dosel; además, el tiempo debe ser suficiente para que lleguen los polinizadores. Como es impredecible saber si uno puede tener éxito en estas observaciones, es más probable que este trabajo pueda ser llevado a cabo por un aficionado o científico que no depende de la carrera. Es imposible proponer a estudiantes un tema como tal para una tesis, por sus resultados impredecibles. De todos modos quiero animar a todos los amantes de la naturaleza para que vayan al campo: necesitamos urgentemente informaciones de este tipo.

Agradecimientos. Quiero agradecer especialmente a Eric Hágsater (México) por su inmensa ayuda en relación a las plantas y por brindarme información sobre las localidades de Stanhopeinae mexicanas, a Gerardo Salazar, Mark Whitten (en orden alfabético) por su discusión al respecto de estas plantas, al Dr. Roman Kaiser (Givaudan Research, Dübendorf, Suiza) por los análisis de lós aromas florales y a mi esposa Corina Gerlach por la ayuda con la correción del español, a mi asistente Jutta Babczinsky por la ayuda en el laboratorio y la diagramación, y a Bert Klein y su equipo de jardineros por cultivar nuestras plantas preciosas.

\section{LiTERATURA CITADA}

Gerlach, G. 1999. 854. Stanhopea Frost ex Hook. 1829(333). En Schlechter: Die Orchideen III / A, Berlin 2355-2372. Jenny, R. 2003. The Genus Stanhopea $1^{\text {th }}$ Part - S. anfracta to $S$. napoensis. Caesiana 22: 1-145.

Jenny, R. 2004. The Genus Stanhopea $2^{\text {nd }}$ Part. Caesiana 22: 146-291.

Rosillo de Velasco, S. 1984. Stanhopea novogaliciana Rosillo, una nueva especie del occidente de Mexico. Orquidea (Méx.) 9(2): 251-260.

Salazar, G.A. \& Soto Arenas, M.A. 2001. A new species of Stanhopea (Orchidaceae) from Mexico. Lindleyana 16: 144-148.

Soto Arenas, M.A. 2003. Stanhopea dodsoniana Soto Arenas \& Salazar. Icon. Orchid. 5-6: t. 673.

Soto Arenas, M.A. 2003. Stanhopea hernandezii (Kunth) Schltr. Icon. Orchid. 5-6: t. 674.

Soto Arenas, M.A. 2003. Stanhopea maculosa Knowles \& Westc. Icon. Orchid. 5-6: t. 675.

Soto Arenas, M.A. 2003. Stanhopea martiana Bateman ex Lindl. Icon. Orchid. 5-6: t. 676.

Soto Arenas, M.A. 2003. Stanhopea oculata (G. Lodd.) Lindl. Icon. Orchid. 5-6: t. 677.

Soto Arenas, M.A. 2003. Stanhopea tigrina Bateman. Icon. Orchid. 5-6: t. 678.

Soto Arenas, M., Salazar, G. \& Gerlach, G. 2003. Stanhopea whittenii Soto Arenas, Salazar \& G.Gerlach. Icon. Orchid. 5-6: t. 679.

Whitten, W.M. \& N.H. Williams 1992. Floral fragrances of Stanhopea (Orchidaceae). Lindleyana 7(3): 130-153. 